technology; with $63.3 \%$ using the internet occasionally, $73.3 \%$ having a cell phone, and $66.7 \%$ accessing the internet using a mobile handheld device. Participants expressed an interest in measuring blood pressure, accessing health information, and managing medication on their cellphone or tablet. Twenty-five percent of participants self-reported not being confident at all in managing their medication without help and remembering to take their medication as prescribed. Fewer participants (54.2\%) were very confident with using technology to help manage medication and treatment. DISCUSSION/ SIGNIFICANCE OF IMPACT: This study provides important insights on the use of technology for managing hypertension in an urban low-income community. Results suggest that medication adherence, the use of technology, and the willingness to use mobile applications for hypertension management is high within this lowincome community. This study gives future researchers a framework on how to assess these specific areas in their own population.

\section{Factors Associated with Hesitancy of Human Papillomavirus Vaccination Among Latina Immigrant Mothers Despite Physician Recommendation}

Alexandra B Khodadadi ${ }^{1}$, David Redden and Isabel Scarinci ${ }^{1}$ University of Alabama at Birmingham

OBJECTIVES/SPECIFIC AIMS: The purpose of this study was to examine factors associated with Latina immigrant mothers' hesitancy in having their 9-12 year old daughters vaccinated against HPV despite a physician recommendation. METHODS/STUDY POPULATION: The data analyzed for this study was from a previous effort that examined the efficacy of an intervention to promote HPV vaccination among daughters (9-12 years of age) of Latina immigrants $(\mathrm{N}=317)$ through a randomized trial. Baseline data among Latina immigrant mothers with unvaccinated daughters was collected prior to this intervention. Participants were surveyed on sociodemographic characteristics, knowledge and perceptions of cervical cancer and HPV, and intention to vaccinate their daughters with the HPV vaccine if recommended by the daughter's physician. RESULTS/ANTICIPATED RESULTS: Out of 317 participants, 205 reported willingness to vaccinate following a physician recommendation $(64.7 \%)$ while 112 reported hesitancy (defined as an answer of "maybe") to vaccinate their daughters (35.3\%). None of the participants indicated "no". No sociodemographic factors were significantly associated with vaccine hesitancy except for the daughter's health insurance status $(p=0.03)$. A significant difference existed in the mother's perceived risk of cervical cancer in herself based on hesitancy and willingness to vaccination $(p<0.001)$. Mother's awareness of HPV ( $p<0.0001)$, knowledge of HPV ( $p<0.01)$, her perceived risk of HPV infection in herself $(p<0.01)$ and in her daughter $(\mathrm{p}<0.0001)$, and her worry about her daughter being infected with HPV $(p=0.02)$ were also significant. Finally, there were differences among mothers in confidence of being able to have their daughters complete all three doses of the vaccine $(p<0.0001)$, and having the time $(\mathrm{p}<0.0001)$ and the money $(\mathrm{p}<0.0001)$ to complete the vaccination series. Factors that were significant in the univariate analysis were then incorporated into a binary logistic multivariable regression. HPV knowledge score was excluded from this analysis due to its limited sample size $(n=169)$. The five variables that were strongly associated with the outcome of vaccine hesitancy included: HPV awareness, mother's perceived risk of HPV infection in their daughter, confidence in the ability to receive all three shots in vaccine series, confidence in being able to afford the vaccine series, and daughter's health insurance. All were positively correlated with intention to vaccinate except for daughter's health insurance status. DISCUSSION/SIGNIFICANCE OF IMPACT: Despite research showing Latinos are pro-vaccination and the association between HCP provider recommendation and vaccine acceptability among parents, over $35 \%$ of Latina immigrant mothers in our study were still hesitant about having their daughters vaccinated against HPV following a physician recommendation. Factors that strongly contributed to this hesitancy included HPV awareness, mother's perceived risk of their daughter being infected with HPV, self-efficacy (ability to receive all required shots and finding time to complete the HPV vaccination series), and daughter's health insurance status. These results indicate that HCP recommendation may be not enough of a driving factor to motivate Latina immigrant mothers to vaccinate their daughters against HPV. Further research efforts should focus on heightening perceived risk of HPV infection, improving knowledge of HPV, and boosting their self-efficacy to get their children vaccinated against HPV.

3113

\section{Formative Evaluation of a Safety Baby Shower Intervention for Rural African American Parents and Community Advisors \\ Rosemary Nabaweesi ${ }^{1}$, Mary Aitken, Samantha H. Mullins, Keneshia Bryant-Moore, Geoffrey M. Curran and Zenobia Harris ${ }^{1}$ University of Arkansas Translational Research Institute}

OBJECTIVES/SPECIFIC AIMS: To explore rural African American parents' and their community advisors' perspectives on the Safety Baby Shower's acceptability, feasibility, and adaptability. METHODS/ STUDY POPULATION: Collaborating with a local community organization, we explored community advisors' and expectant women's SBS experiences to understand intervention delivery and adoption in a rural underserved community (RUC). The Consolidated Framework for Implementation Research guided our data collection and analysis using focus groups and key informant interviews. We used directed content analysis to generate themes and sub codes. RESULTS/ANTICIPATED RESULTS: Five focus groups (21 participants) and one key informant interview were conducted. Identified barriers that hinder feasibility and acceptability included resources, time/ flexibility, intervention location, cultural norms and beliefs, and the lack of a birthing hospital in the county. "Baby proofing", "reinforcement products" and "teaching sleep safety on the same day as infant clinical appointment" are expectant mothers' exemplars for what comes to their minds when asked to think about safety baby showers. To improve feasibility, both community advisors and expectant mothers suggested adaptations ranging from decentralizing or rotating intervention location, using different delivery sites such as churches, scheduling intervention outside business hours, to incorporating intervention into school health fairs and barbeque events. Social media emerged as a facilitator, and integrating safe sleep education into personal baby showers emerged as an implementation strategy. DISCUSSION/SIGNIFICANCE OF IMPACT: The community advisors and expectant mothers identified a wide spectrum of potential adaptations that have potential to improve safe sleep knowledge and practices. In the next study phase, identified themes will inform intervention adaptation and suggested implementation strategies will support uptake of the adapted SBS. Identifying transformative implementation strategies and 